\title{
Revisión Sistemática: Los recordatorios a pacientes mejoran las tasas de inmunización
}

Effect of Patient Reminder/Recall Interventions on Immunization Rates. Szilagyi PG, Bordley C, Chelminski A. JAMA2000:1820-1827.

\begin{abstract}
Objetivo
Valorar la efectividad de los recordatorios, y sus diferentes tipos, para mejorar las tasas de inmunización en diferentes poblaciones
\end{abstract} y ámbitos.

\section{Fuentes de datos}

Búsqueda en MEDLINE, EMBASE, PsychINFO, Sociological Abstracts, CAB Health Abstracts y principales referencias (artículos, resúmenes, publicaciones de conferencias y archivos de colaboradores de estudios).

\section{Selección de estudios}

Estudios en inglés (hasta 1998) de recordatorios a pacientes que midieran tasas de inmunizaciones recomendadas (ensayos controlados y aleatorizados, estudios antes-después*) siguiendo los criterios de revisión de la Colaboración Cochrane.1-2 Los recordatorios a médicos no fueron evaluados a menos que fueran en combinación con los de los pacientes, en cuyo caso se analizaron separadamente.

\section{Extracción de datos}

Fueron elegibles 41 de 109 estudios identificados. Los estudios eran evaluados independientemente por dos revisores mediante un protocolo (Cochrane ) y las discrepancias resueltas formalmente por consenso. Se evaluaron numerosos criterios de calidad.

\section{Resultados principales}

Los recordatorios mejoraron las tasas de inmunización en 33/41 estudios (80\%), independientemente de la tasa de inmunización basal, edad del paciente, ámbito o década de realización del estudio o tipo de vacuna. El aumento de las tasas fue de entre el 5 y $20 \%$. Todos los tipos de recordatorios fueron efectivos: envío de postal, de carta, telefónico (el más efectivo y costoso) o programas computarizados de autodiscado, y su beneficio varió según la aplicación de la intervención:

\begin{tabular}{l|l|l|l}
\hline $\begin{array}{l}\text { Intervención } \\
\text { (N0 de estudios) }\end{array}$ & $\begin{array}{l}\text { Mejora } \\
\% \text { \# }\end{array}$ & OR & IC95\% \\
\hline Inmunización niños (15) & $6-34 \%$ & 2,02 & $1,49-2,72$ \\
\hline Antigripal niños (2) & $>20 \%$ & 4,25 & $2,10-8,60$ \\
\hline $\begin{array}{l}\text { Antineumocóccica 0 } \\
\text { Antitetánica Adultos (7) }\end{array}$ & $4-27 \%$ & 5,14 & $1,21-21,8$ \\
\hline Antigripal Adultos (21) & $>20 \%$ & 2,29 & $1,69-3,10$ \\
\hline Centro académico (16) & $3,4-21 \% \&$ & 3,33 & $1,98-5,58$ \\
\hline Centro Privado (17) & $8,2-21 \% \&$ & 1,79 & $1,14-2,22$ \\
\hline Centro Público (10) & $3,4-14 \% \&$ & 2,09 & $1,42-3,07$ \\
\hline
\end{tabular}

\# \% de mejora en valores absolutos de los estudios positivos \& Medianas de cambio por subtipo de Centro

\begin{tabular}{l|l}
$\begin{array}{l}\text { Tipo de recordatorio } \\
\text { (NO de estudios) }\end{array}$ & OR (IC95\%) \\
\hline Postal (8) & $1,91(1,27-2,28)$ \\
\hline Carta (23) & $2,57(1,83-3,59)$ \\
\hline Telefónico (8) & $5,52(3,90-7,79)$ \\
\hline Autodiscado (4) & $1,51(1,18-1,93)$ \\
\hline Postal + Carta (1) & $1,81(1,11-2,95)$ \\
\hline Con Seguimiento (2) & $3,42(0,88-13,3)$ \\
\hline Todos los sistemas (38) & $2,50(2,00-3,13)$ \\
\hline A paciente y Médico (8) & $3,16(2,10-4,75)$ \\
\hline
\end{tabular}

\section{Conclusiones}

Los recordatorios a pacientes en ámbitos de atención primaria mejoran las tasas de inmunización.

\section{Comentario}

Aún en países como EE.UU. donde las tasas de inmunización están en aumento, los niveles de cobertura no cumplen con las metas nacionales. Por ejemplo en 1997 el promedio nacional de cobertura infantil fue de $65,4 \% ; 3$ la vacunación antigripal en $<65$ años de alto riesgo fue $<30 \%$, lejos de la meta del $60 \%$ para el 2000; y aunque la cobertura antigripal mejoró, no está homogéneamente distribuida. Es más baja en blancos e hispanos, ${ }^{4}$ y cualquier inmunización en general es más baja en poblaciones de bajos recursos. $5 \mathrm{Si}$ bien carecemos de datos nacionales en Argentina, la epidemia de sarampión 98-99 indica claramente la insuficiente cobertura alcanzada.

Los distintos sistemas recordatorios evaluados en esta revisión se mostraron efectivos para todas las vacunas, poblaciones o ámbitos en que fueron estudiados. Los telefónicos y los intensivos son los más efectivos y costosos. Para seleccionar una estrategia apropiada, la costo-efectividad (costo por cada paciente adicional vacunado) puede orientarnos en la toma de decisiones. Aunque fue medida en pocos estudios, la costo-efectividad rondó los $\$ 10 /$ paciente, pero en los más intensivos (seguimiento anual) trepó a \$316 anuales/niño extra adecuadamente vacunado.
Muchos estudios detectaron un aumento de prácticas preventivas como efecto secundario al recuerdo de inmunizaciones lo que, indirectamente, aumentaría los beneficios de la intervención.

Una de las limitaciones de este estudio es que sólo se incluyeron estudios en inglés, si bien en los nueve estudios de países que no incluyen EE.UU. tuvieron todos resultados positivos. Además el sesgo de publicación (sólo suelen publicarse los estudios de resultados positivos) se intentó minimizar mediante una extensa búsqueda y no fue detectado en el análisis estadístico. Otra limitación, para la combinación de datos, fue una esperable heterogeneidad de resultados dadas las distintas poblaciones y ámbitos estudiados durante un período de 30 años. Los hallazgos de esta revisión sistemática apoyan la recomendación del uso de recordatorios por parte de todo médico de atención primaria para mejorar los niveles de cobertura de inmunizaciones de la población a su cargo. Especialmente dada la complejidad creciente de los esquemas y las mayores expectativas de los pacientes y los sistemas sobre el desempeño de dichos médicos. Serían muy útiles nuevas investigaciones focalizadas en la implementación de esta eficaz estrategia.

\section{Dr. Agustín Ciapponi [ Unidad de Medicina Familiar y Preventiva. Hospital Italiano de Buenos Aires ]}

\section{Referencias}

1. Ortiz Z. Editorial: La colaboración Cochrane. Evidencia en Atención Primaria. 2000 Mayo-Junio Vol 3 Nro 3:66-67.

2. Clark M, Oxman AD, eds. Cochrane Reviwers' Handbook 4.0 In Review Manager [computer program]. Version 4.0 Oxford England: The Cochrane Collaboration; 1999. http://www.cochrane.dk/cochrane/handbook/handbook.htm

3. Selected National Averages. Adapted from NCQA's State of Managed Care Quality. NCQANews http://www.ncqa.org/Pages/Communications/News/99avgs.htm

4. Prevention and control of influenza: Recommendations of the Advisory Committee on Immunization Practices (ACIP). MMWR-Morb-Mortal-Wkly-Rep. 2000 Apr 14; 49(RR03): 1-38 5. Centers of Disease Control and Prevention. Vaccination coverage by race/ethnicity and poverty level among children aged 19-35 months-Unites States. MMWR-Morb-Mortal-Wkly-Rep. 1997; 47:956-959. 